\title{
Ranking Of Octagonal Intuitionistic Fuzzy Numbers
}

\author{
G.Menaka \\ Assistant Professor, Department of Mathematics, Sri Shakthi Institute of Engineering and Technology, \\ Coimbatore, India
}

\begin{abstract}
In This Paper We Introduce Octagonal Intuitionistic Fuzzy Numbers With Its Membership And NonMembership Functions. A New Method Is Proposed For Finding An Optimal Solution For Intuitionistic Fuzzy Transportation Problem, In Which The Supplies And Demands Are Octagonal Intuitionistic Fuzzy Numbers. The Procedure Is Illustrated With A Numerical Example.

Keywords: Intuitionistic Fuzzy Transportation Problems, Initial Basic Feasible Solution, Modi Method, Ranking Method, Octagonal Intuitionistic Fuzzy Numbers, Optimal Solution.
\end{abstract}

\section{Introduction}

The central concept in the problem is to find the least total transportation cost of commodity. In general, transportation problems are solved with assumptions that the cost, supply and demand are specified in precise manner. However in many cases the decision maker has no precise information about the coefficient belonging to the transportation problem. Intuitionistic fuzzy set is a powerful tool to deal with such vagueness.

The concept of Intuitionistic Fuzzy Sets (IFSs), proposed by Atanassov in [1]and[2], has been found to be highly useful to deal with vagueness. Many authors discussed the solutions of Fuzzy Transportation Problem (FTP) using various techniques. In 1982, O'heigeartaigh [9] proposed an algorithm to solve Fuzzy Transportation Problem with triangular membership function. In 2013, Nagoor Gani.A and Abbas. S [8], introduced an new method for solving in Fuzzy Transportation Problem. In 2016, Mrs. Kasthuri. B [7] introduced Pentagonal intuitionistic fuzzy. In 2015, A. Thamaraiselvi and R. Santhi [3] introduced Hexagonal Intuitionistic Fuzzy Numbers. In 2015, Thangaraj Beaula - M. Priyadharshini [4] proposed. A New Algorithm for Finding a Fuzzy Optimal Solution.K. Prasanna Devi, M. Devi Durga [5] and G. Gokila, Juno Saju [6] introduced Octagonal Fuzzy Number.

The paper is organized as follows, In section 2, introduction with some basic concepts of Intuitionistic fuzzy numbers, In section 3, introduce Octagonal Intuitionistic Fuzzy Definition and proposed algorithm followed by a Numerical example using Modi method and In section 4, finally the paper is concluded.

\section{II.1. FUZZY SET [FS][3]:}

\section{Preliminaries}

Let $\mathrm{X}$ be a nonempty set. A fuzzy set $\bar{A}$ of Xis defined as $\bar{A}=\left\{\left\langle x, \mu_{\bar{A}}(\mathrm{x})\right\rangle / x \in X\right\}$. Where $\mu_{\bar{A}}(\mathrm{x})$ is called membership function, which maps each element of $\mathrm{X}$ to a value between 0 and 1 .

\section{II.2. FUZZY NUMBER [FN][3]:}

A fuzzy number is a generalization of a regular real number and which does not refer to a single value but rather to a connected a set of possible values, where each possible value has its weight between 0 and 1 . The weight is called the membership function.

A fuzzy number $\bar{A}$ is a convex normalized fuzzy set on the real line $\mathrm{R}$ such that

- There exist at least one $\mathrm{x} \in \mathrm{R}$ with $\mu_{\bar{A}}(\mathrm{x})=1$.

- $\mu_{\bar{A}}(\mathrm{x})$ Is piecewise continuous.

\section{II.3. TRIANGULAR FUZZY NUMBER [TFN] [3]:}

A Triangular fuzzy number $\bar{A}$ is denoted by 3 - tuples $\left(a_{1}, a_{2}, a_{3}\right)$, where $a_{1}, a_{2}$ and $a_{3}$ are real numbers and $a_{1} \leq a_{2} \leq a_{3}$ with membership function defined as

$$
\mu_{\bar{A}}(\mathrm{x})=\left\{\begin{array}{cr}
\frac{x-a_{1}}{a_{2}-a_{1}} & \text { for } a_{1} \leq x \leq a_{2} \\
\frac{a_{3}-x}{a_{3}-a_{2}} & \text { for } a_{2} \leq x \leq a_{3} \\
0 & \text { otherwise }
\end{array}\right\}
$$




\section{II.4. TRAPEZOIDAL FUZZY NUMBER [TRFN] [3]:}

A trapezoidal Fuzzy number is denoted by 4 tuples $\bar{A}=\left(a_{1}, a_{2}, a_{3}, a_{4}\right)$, Where $a_{1}, a_{2}, a_{3}$ and $a_{4}$ are real numbers and $a_{1} \leq a_{2} \leq a_{3} \leq a_{4}$ with membership function defined as

$$
\mu_{\bar{A}}(\mathrm{x})=\left\{\begin{array}{cc}
\frac{x-a_{1}}{a_{2}-a_{1}} & \text { for } a_{1} \leq x \leq a_{2} \\
1 & \text { for } a_{2} \leq x \leq a_{3} \\
\frac{a_{4}-x}{a_{4}-a_{3}} & \text { for } a_{3} \leq x \leq a_{4} \\
0 & \text { otherwise }
\end{array}\right\}
$$

\section{II.5. PENTAGON FUZZY NUMBER [PFN] [3]:}

A Pentagon Fuzzy Number $\bar{A}_{P}=\left(a_{1}, a_{2}, a_{3}, a_{4}, a_{5}\right)$. Where $a_{1}, a_{2}, a_{3}, a_{4}$ and $a_{5}$ are real numbers and $a_{1} \leq a_{2} \leq a_{3} \leq a_{4} \leq a_{5}$ with membership function is given below

$$
\mu_{\bar{A}}(\mathrm{x})=\left\{\begin{array}{cc}
\frac{x-a_{1}}{a_{2}-a_{1}} & \text { for } a_{1} \leq x \leq a_{2} \\
\frac{x-a_{2}}{a_{3}-a_{2}} & \text { for } a_{2} \leq x \leq a_{3} \\
1 & \text { for } x=a_{3} \\
& \frac{a_{4}-x}{a_{4}-a_{3}} \\
& \text { for } a_{3} \leq x \leq a_{4} \\
\frac{a_{5}-x}{a_{5}-a_{4}} & \text { for } a_{4} \leq x \leq a_{5} \\
0 & \text { for } x>a_{5}
\end{array}\right\}
$$

\section{II.6. HEXAGONAL FUZZY NUMBER [HFN] [3]:}

A Hexagon Fuzzy Number $\bar{A}_{H}$ is specified by 6 tuples, $\bar{A}_{H}=\left(a_{1}, a_{2}, a_{3}, a_{4}, a_{5}, a_{6}\right)$. Where $a_{1}, a_{2}, a_{3}, a_{4}, a_{5}$ and $a_{6}$ are real numbers and $a_{1} \leq a_{2} \leq a_{3} \leq a_{4} \leq a_{5} \leq a_{6}$ with membership function is given below,

$$
\mu_{\bar{A}}(\mathrm{x})=\left\{\begin{array}{ll}
\frac{1}{2}\left(\frac{x-a_{1}}{a_{2}-a_{1}}\right) & \text { for } a_{1} \leq x \leq a_{2} \\
\frac{1}{2}+\frac{1}{2}\left(\frac{x-a_{2}}{a_{3}-a_{2}}\right) & \text { for } a_{2} \leq x \leq a_{3} \\
1 & \text { for } a_{3} \leq x \leq a_{4} \\
1-\frac{1}{2}\left(\frac{x-a_{4}}{a_{5}-a_{4}}\right) & \text { for } a_{4} \leq x \leq a_{5} \\
\frac{1}{2}\left(\frac{a_{6}-x}{a_{6}-a_{5}}\right) & \text { for } a_{5} \leq x \leq a_{6} \\
0 & \text { for } \text { otherwise }
\end{array}\right\}
$$

\section{II.7. OCTAGONAL FUZZY NUMBER [OFN] [4]:}

A Fuzzy Number $\bar{A}_{O C}$ is a normal Octagonal Fuzzy Number denoted by $\bar{A}=\left(a_{1}, a_{2}, a_{3}, a_{4}, a_{5}, a_{6}, a_{7}, a_{8}\right)$. where $a_{1}, a_{2}, a_{3}, a_{4}, a_{5}, a_{6}, a_{7}$ and $a_{8}$ are real numbers and its membership function $\mu_{\bar{A}}(\mathrm{x})$ is given below: 
Where $0<\mathrm{k}<1$.

$$
\mu_{\bar{A}}(\mathrm{x})=\left\{\begin{array}{lr}
0 & \text { for } \quad x<a_{1} \\
k\left(\frac{x-a_{1}}{a_{2}-a_{1}}\right) & \text { for } a_{1} \leq x \leq a_{2} \\
k & \text { for } a_{2} \leq x \leq a_{3} \\
k+(1-k)\left(\frac{x-a_{3}}{a_{4}-a_{3}}\right) & \text { for } a_{3} \leq x \leq a_{4} \\
1 & \text { for } a_{4} \leq x \leq a_{5} \\
k+(1-k)\left(\frac{a_{6}-x}{a_{6}-a_{5}}\right) & \text { for } a_{5} \leq x \leq a_{6} \\
k & \text { for } a_{6} \leq x \leq a_{7} \\
k\left(\frac{a_{8}-x}{a_{8}-a_{7}}\right) & \text { for } a_{7} \leq x \leq a_{8} \\
0 & \text { for } x>a_{8}
\end{array}\right\}
$$

\section{II.8. INTUITIONISTIC FUZZY SET [IFS] [3]:}

Let $\mathrm{X}$ is a non-empty set. An Intuitionistic fuzzy set $\bar{A}^{I}$ of $\mathrm{X}$ is defined as,

$\bar{A}^{I}=\left\{\left\langle\mathrm{x}, \mu_{\bar{A}}(x), \vartheta_{\bar{A}^{I}}(\mathrm{x})\right\rangle / \mathrm{x} \in X\right\}$. Where $\mu_{\bar{A}^{I}}(x)$ and $\vartheta_{\bar{A}}(\mathrm{x})$ are membership and non-membership function. Such that $\mu_{\bar{A}^{I}}(x), \vartheta_{\bar{A}^{I}}(\mathrm{x}): \mathrm{X} \rightarrow[0,1]$ and $0 \leq \mu_{\bar{A}^{I}}(x)+\vartheta_{\bar{A}^{I}}(\mathrm{x}) \leq 1$ for all $\mathrm{x} \in X$.

\section{II.9. INTUITIONISTIC FUZZY NUMBER [IFN] [3]:}

An Intuitionistic Fuzzy Subset $\bar{A}^{I}=\left\{\left\langle\mathrm{x}, \mu_{\bar{A}^{I}}(x), \vartheta_{\bar{A}^{I}}(\mathrm{x})\right\rangle / \mathrm{x} \in X\right\}$ of the real line $\mathrm{R}$ is called an Intuitionistic Fuzzy Number, if the following conditions hold,

- $\quad$ There exists $\mathrm{m} \in \mathrm{R}$ suct that $\mu_{\bar{A}^{I}}(\mathrm{~m})=1$ and $\vartheta_{\bar{A}^{I}}(\mathrm{~m})=0$.

- $\quad \mu_{\bar{A}^{I}}$ is a continuous function from $\mathrm{R} \rightarrow[0,1]$ such that

- $0 \leq \mu_{\bar{A}^{I}}(x)+\vartheta_{\bar{A}^{I}}(\mathrm{x}) \leq 1$ for all $\mathrm{x} \in X$.

The membership and non- membership functions of $\bar{A}^{I}$ are in the following form

$$
\begin{aligned}
& \mu_{\bar{A}^{I}}(x)=\left\{\begin{array}{cc}
0 & \text { for }-\infty<x \leq a_{1} \\
f(x) & \text { for } a_{1} \leq x \leq a_{2} \\
1 & \text { for } \\
g(x) & \text { for } a_{2} \leq x \leq a_{2} \\
0 & \text { for } a_{3} \leq x<\infty
\end{array}\right\} \\
& \vartheta_{\bar{A}^{I}}(\mathrm{x})=\left\{\begin{array}{ccc}
1 & \text { for }-\infty<x \leq a_{1}{ }^{\prime} \\
f^{\prime}(x) & \text { for } a_{1}{ }^{\prime} \leq x \leq a_{2} \\
0 & \text { for } \quad x=a_{2} \\
g^{\prime}(x) & \text { for } a_{2} \leq x \leq a_{3}{ }^{\prime} \\
1 & \text { for } a_{3} \leq x<\infty
\end{array}\right\}
\end{aligned}
$$

Where $f, f^{\prime}, g, g^{\prime}$ are functions from $\mathrm{R} \rightarrow[0,1] . f$ and $g^{\prime}$ are strictly increasing functions and $g$ and $f^{\prime}$ are strictly decreasing functions with the conditions $0 \leq f(x)+f^{\prime}(x) \leq 1$ and $0 \leq g(x)+g^{\prime}(x) \leq 1$.

\section{II.10. TRIANGULAR INTUITIONISTIC FUZZY NUMBERS [TFIN [3]:}

A Triangular Intuitionistic Fuzzy Number $\bar{A}^{I}$ is denoted by $\bar{A}^{I}=\left(a_{1}, a_{2}, a_{3}\right)\left(a_{1}^{\prime}, a_{2}, a_{3}^{\prime}\right)$. Where $a_{1}^{\prime} \leq a_{1} \leq$ $a_{2} \leq a_{3} \leq a_{3}^{\prime}$ with the following membership $\mu_{\bar{A}^{I}}(x)$ and non membership fuuntion $\vartheta_{\bar{A}}(\mathrm{x})$.

$$
\begin{aligned}
& \mu_{\bar{A}^{I}}(x)=\left\{\begin{array}{lr}
\frac{x-a_{1}}{a_{2}-a_{1}} & \text { for } a_{1} \leq x \leq a_{2} \\
\frac{a_{3}-x}{a_{3}-a_{2}} & \text { for } a_{2} \leq x \leq a_{3} \\
0 & \text { otherwise }
\end{array}\right\} \\
& \vartheta_{\bar{A}^{I}}(\mathrm{x})=\left\{\begin{array}{cc}
\frac{a_{2-x}}{a_{2}-a_{1^{\prime}}} & \text { for } a_{1}{ }^{\prime} \leq x \leq a_{2} \\
\frac{x-a_{2}}{a_{3^{\prime}-a_{2}}} & \text { for } a_{2} \leq x \leq a_{3}{ }^{\prime}
\end{array}\right\}
\end{aligned}
$$




\section{II.11. TRAPEZOIDAL INTUITIONISTIC FUZZY NUMBERS [TRIFN] [3]:}

A Trapezoidal Intuitionistic Fuzzy Number is denoted by $\bar{A}^{I}=$ $\left(a_{1}, a_{2}, a_{3}, a_{4}\right),\left(a_{1}^{\prime}, a_{2}, a_{3}, a_{4}{ }^{\prime}\right)$. Where $a_{1}{ }^{\prime} \leq a_{1} \leq a_{2} \leq a_{3} \leq a_{4} \leq a_{4}{ }^{\prime}$ with membership and non membership functions are defined as follows

$$
\begin{gathered}
\mu_{\bar{A}^{I}}(x)=\left\{\begin{array}{lr}
\frac{x-a_{1}}{a_{2}-a_{1}} & \text { for } a_{1} \leq x \leq a_{2} \\
1 & \text { for } a_{2} \leq x \leq a_{3} \\
\frac{a_{4}-x}{a_{4}-a_{3}} & \text { for } a_{3} \leq x \leq a_{4} \\
0 & \text { otherwise }
\end{array}\right\} \\
\vartheta_{\bar{A}^{I}}(\mathrm{x})=\left\{\begin{array}{ll}
\frac{x-a_{1}{ }^{\prime}}{a_{2}-a_{1}{ }^{\prime}} & \text { for } a_{1}{ }^{\prime} \leq x \leq a_{2} \\
1 & \text { for } a_{2} \leq x \leq a_{3} \\
\frac{a_{4}{ }^{\prime}-x}{a_{4}{ }^{\prime}-a_{3}} & \text { for } a_{3} \leq x \leq a_{4}{ }^{\prime} \\
0 & \text { otherwise }
\end{array}\right\}
\end{gathered}
$$

\section{II.12. PENTAGONAL INTUITIONISTIC FUZZY NUMBER [PIFN][3]:}

A Pentagonal Intuitionistic Fuzzy Number $\bar{A}^{I}$ is defined as $\bar{A}^{I}=\left\{\left(a_{1}, b_{1}, c_{1}, d_{1}, e_{1}\right)\left(a_{2}, b_{2}, c_{2}, d_{2}, e_{2}\right)\right\}$. Where all $a_{1}, b_{1}, c_{1}, d_{1}, e_{1}, a_{2}, b_{2}, c_{2}, d_{2}, e_{2}$ are real numbers and its membership function $\mu_{\bar{A}^{I}}(x)$, non membership function $\vartheta_{\bar{A}^{I}}(\mathrm{x})$ are given by

$$
\begin{gathered}
\mu_{\bar{A}^{I}}(x)=\left\{\begin{array}{ccr}
0 & \text { for } & x<a_{1} \\
\frac{x-a_{1}}{b_{1}-a_{1}} & \text { for } a_{1} \leq x \leq b_{1} \\
\frac{x-b_{1}}{c_{1}-b_{1}} & \text { for } b_{1} \leq x \leq c_{1} \\
1 & \text { for } & x=c_{1} \\
\frac{d_{1}-x}{d_{1}-c_{1}} & \text { for } c_{1} \leq x \leq d_{1} \\
\frac{e_{1}-x}{e_{1}-d_{1}} & \text { fr } d_{1} \leq x \leq e_{1} \\
0 & \text { for } \quad x>e_{1}
\end{array}\right\} \\
\vartheta_{\bar{A}^{I}}(\mathrm{x})=\left\{\begin{array}{ccc}
1 & \text { for } & x<a_{2} \\
\frac{b_{2}-x}{b_{2}-a_{2}} & \text { for } a_{2} \leq x \leq b_{2} \\
\frac{c_{2}-x}{c_{2}-b_{2}} & \text { for } b_{2} \leq x \leq c_{2} \\
\frac{x-c_{2}}{d_{2}-c_{2}} & \text { for } & x=c_{1} \\
\frac{x-d_{2}}{e_{2}-d_{2}} & \text { for } c_{2} \leq x \leq d_{2} \\
1 & \text { for } d_{2} \leq x \leq e_{2}
\end{array}\right\}
\end{gathered}
$$

\section{II.13. HEXAGONAL INTUITIONISTIC FUZZY NUMBER [HIFN] [3]:}

A Hexagonal Intuitionistic Fuzzy Number is specified by

$\bar{A}_{H}{ }^{I}=\left(a_{1}, a_{2}, a_{3}, a_{4}, a_{5}, a_{6}\right),\left(a_{1}{ }^{\prime}, a_{2}{ }^{\prime}, a_{3}, a_{4}, a_{5}{ }^{\prime}, a_{6}{ }^{\prime}\right)$ Where $a_{1}, a_{2}, a_{3}, a_{4}, a_{5}, a_{6}, a_{1}^{\prime}, a_{2}^{\prime}, a_{5}^{\prime}$ and $a_{6}^{\prime}$ are real numbers such that $a_{1}{ }^{\prime} \leq a_{1} \leq a_{2}{ }^{\prime} \leq a_{2} \leq a_{3} \leq a_{4} \leq a_{5} \leq a_{5}{ }^{\prime} \leq a_{6} \leq a_{6}{ }^{\prime}$ and its membership and nonmembership functions are given below 


$$
\begin{gathered}
\mu_{\bar{A}^{I}} \quad(x)=\left\{\begin{array}{cc}
\frac{1}{2}\left(\frac{x-a_{1}}{a_{2}-a_{1}}\right) & \text { for } a_{1} \leq x \leq a_{2} \\
\frac{1}{2}+\frac{1}{2}\left(\frac{x-a_{2}}{a_{3}-a_{2}}\right) & \text { for } a_{2} \leq x \leq a_{3} \\
1 & \text { for } a_{3} \leq x \leq a_{4} \\
1-\frac{1}{2}\left(\frac{x-a_{4}}{a_{5}-a_{4}}\right) & \text { for } a_{4} \leq x \leq a_{5} \\
\frac{1}{2}\left(\frac{a_{6}-x}{a_{6}-a_{5}}\right) & \text { for } a_{5} \leq x \leq a_{6} \\
0 & \text { for } \text { otherwise }
\end{array}\right\} \\
\vartheta_{\bar{A}^{I}}(\mathrm{x})=\left\{\begin{array}{cc}
1-\frac{1}{2}\left(\frac{x-a_{1}{ }^{\prime}}{a_{2}{ }^{\prime}-a_{1}{ }^{\prime}}\right) & \text { for } a_{1}{ }^{\prime} \leq x \leq a_{2}{ }^{\prime} \\
\frac{1}{2}\left(\frac{a_{3}-x}{a_{3}-a_{2}{ }^{\prime}}\right) & \text { for } a_{2}{ }^{\prime} \leq x \leq a_{3} \\
0 & \text { for } a_{3} \leq x \leq a_{4} \\
\frac{1}{2}\left(\frac{x-a_{4}}{a_{5}{ }^{\prime}-a_{4}}\right) & \text { for } a_{4} \leq x \leq a_{5}{ }^{\prime} \\
\frac{1}{2}+\frac{1}{2}\left(\frac{x-a_{5}{ }^{\prime}}{a_{6}{ }^{\prime}-a_{5}{ }^{\prime}}\right) & \text { for } a_{5}{ }^{\prime} \leq x \leq a_{6}{ }^{\prime} \\
1 & \text { for } \text { otherwise }
\end{array}\right\}
\end{gathered}
$$

\section{Octagonal Intuitionistic Fuzzy Number}

\section{III.1. OCTAGONAL INTUITIONISTIC FUZZY NUMBER [OIFN]}

An Octagonal Intuitionistic Fuzzy Number is specified by $\bar{A}_{o c}{ }^{I}=\left(a_{1}, a_{2}, a_{3}, a_{4}, a_{5}, a_{6}, a_{7}, a_{8}\right)$, $\left(a_{1}{ }^{\prime}, a_{2}{ }^{\prime}, a_{3}^{\prime}, a_{4}, a_{5}, a_{6}^{\prime}, a_{7}^{\prime}, a_{8}{ }^{\prime}\right)$. Where $a_{1}, a_{2}, a_{3}, a_{4}, a_{5}, a_{6}, a_{7}, a_{8}, a_{1}{ }^{\prime}, a_{2}{ }^{\prime}, a_{3}^{\prime}, a_{6}^{\prime}, a_{7}^{\prime}$ and $a_{8}^{\prime}$ and its membership and non-membership functions are given below

$$
\begin{aligned}
& \mu_{\bar{A}}(\mathrm{x})=\left\{\begin{array}{ll}
0 & \text { for } \quad x<a_{1} \\
k\left(\frac{x-a_{1}}{a_{2}-a_{1}}\right) & \text { for } a_{1} \leq x \leq a_{2} \\
k & \text { for } a_{2} \leq x \leq a_{3} \\
k+(1-k)\left(\frac{x-a_{3}}{a_{4}-a_{3}}\right) & \text { for } a_{3} \leq x \leq a_{4} \\
1 & \text { for } a_{4} \leq x \leq a_{5} \\
k+(1-k)\left(\frac{a_{6}-x}{a_{6}-a_{5}}\right) & \text { for } a_{5} \leq x \leq a_{6} \\
k & \text { for } a_{6} \leq x \leq a_{7} \\
k\left(\frac{a_{8}-x}{a_{8}-a_{7}}\right) & \text { for } a_{7} \leq x \leq a_{8} \\
0 & \text { for } x>a_{8}
\end{array}\right\} \\
& \vartheta_{\bar{A}} I(\mathrm{x})=\left\{\begin{array}{lc}
1 & \text { for } a_{1}^{\prime}<x \\
k+(1-k)\left(\frac{a_{2}{ }^{\prime}-x}{a_{2}{ }^{\prime}-a_{1}{ }^{\prime}}\right) & \text { for } a_{1}{ }^{\prime} \leq x \leq a_{2}{ }^{\prime} \\
k & \text { for } a_{2}{ }^{\prime} \leq x \leq a_{3}{ }^{\prime} \\
k\left(\frac{a_{4}-x}{a_{4}-a_{3}{ }^{\prime}}\right) & \text { for } a_{3}{ }^{\prime} \leq x \leq a_{4} \\
0 & \text { for } a_{4} \leq x \leq a_{5} \\
k\left(\frac{x-a_{5}}{a_{6}{ }^{\prime}-a_{5}}\right) & \text { for } a_{5} \leq x \leq a_{6}{ }^{\prime} \\
k & \text { for } a_{6}{ }^{\prime} \leq x \leq a_{7}{ }^{\prime} \\
k+(1-k)\left(\frac{x-a_{7}{ }^{\prime}}{a_{8}{ }^{\prime}-a_{7}{ }^{\prime}}\right) & \text { for } a_{7}{ }^{\prime} \leq x \leq a_{8}{ }^{\prime} \\
1 & \text { for } x>a_{8}{ }^{\prime}
\end{array}\right\}
\end{aligned}
$$


Graphical representation of Octagonal Intuitionistic Fuzzy Number for k $=0.5$

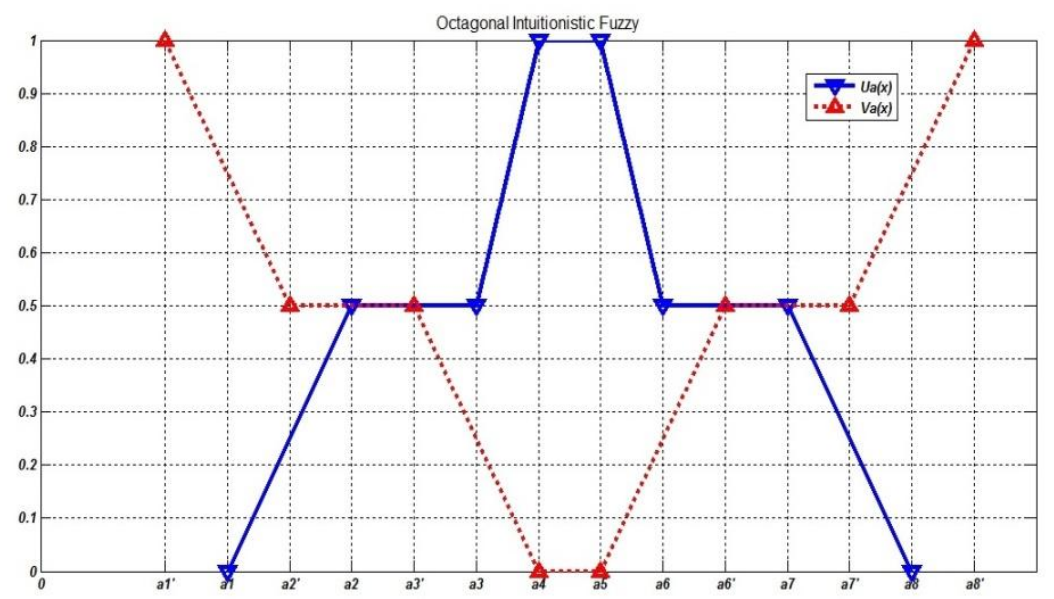

Membership Function $\mu_{\bar{A}}(\mathrm{x})$.

---- Non Membership Function $\vartheta_{\bar{A}^{I}}(\mathrm{x})$.

\section{III.2. ARITHMETIC OPERATIONS ON OCTAGONAL INTUITIONISTIC FUZZY NUMBERS}

Let $\bar{A}_{o c}{ }^{I}=\left(a_{1}, a_{2}, a_{3}, a_{4}, a_{5}, a_{6}, a_{7}, a_{8}\right)\left(a_{1}^{\prime}, a_{2}{ }^{\prime}, a_{3}^{\prime}, a_{4}, a_{5}, a_{6}^{\prime}, a_{7}^{\prime}, a_{8}\right)$ and $\bar{B}_{o c}{ }^{I}=$ $\left(b_{1}, b_{2}, b_{3}, b_{4}, b_{5}, b_{6}, b_{7}, b_{8}\right)\left(b_{1}{ }^{\prime}, b_{2}{ }^{\prime}, b_{3}^{\prime}, b_{4}, b_{5}, b_{6}^{\prime}, b_{7}^{\prime}, b_{8}\right)$ be two Octagonal Intuitionistic Fuzzy Numbers , then the arithmetic operations are as follows.

\section{III.2.1. ADDITION}

$$
\begin{gathered}
\bar{A}_{o c}{ }^{I}+\bar{B}_{o c}{ }^{I}=\left(a_{1}+b_{1}, a_{2}+b_{2}, a_{3}+b_{3}, a_{4}+b_{4}, a_{5}+b_{5}, a_{6}+b_{6}, a_{7}+b_{7}, a_{8}+b_{8}\right) \\
\left(a_{1}^{\prime}+b_{1}^{\prime}, a_{2}^{\prime}+b_{2}^{\prime}, a_{3}^{\prime}+b_{3}^{\prime}, a_{4}+b_{4}, a_{5}+b_{5}, a_{6}{ }^{\prime}+b_{6}^{\prime}, a_{7}^{\prime}+b_{7}^{\prime}, a_{8}^{\prime}+b_{8}\right)
\end{gathered}
$$

\section{III.2.2. SUBTRACTION}

$$
\begin{gathered}
\bar{A}_{o c}{ }^{I}-\bar{B}_{o c}{ }^{I}=\left(a_{1}-b_{8}, a_{2}-b_{7}, a_{3}-b_{6}, a_{4}-b_{5}, a_{5}-b_{4}, a_{6}-b_{3}, a_{7}-b_{2}, a_{8}-b_{1}\right) \\
\left(a_{1}^{\prime}-b_{8}^{\prime}, a_{2}^{\prime}-b_{7}^{\prime}, a_{3}^{\prime}-b_{6}^{\prime}, a_{4}-b_{5}, a_{5}-b_{4}, a_{6}{ }^{\prime}-b_{3}^{\prime}, a_{7}^{\prime}-b_{2}^{\prime}, a_{8}^{\prime}-b_{1}\right) .
\end{gathered}
$$

\section{III.2.3. MULTIPLICATION}

$$
\begin{gathered}
\bar{A}_{o c}{ }^{I} * \bar{B}_{o c}{ }^{I}=\left(a_{1} * b_{1}, a_{2} * b_{2}, a_{3} * b_{3}, a_{4} * b_{4}, a_{5} * b_{5}, a_{6} * b_{6}, a_{7} * b_{7}, a_{8} * b_{8}\right) \\
\left(a_{1}^{\prime} * b_{1}^{\prime}, a_{2}^{\prime} * b_{2}^{\prime}, a_{3}^{\prime} * b_{3}^{\prime}, a_{4} * b_{4}, a_{5} * b_{5}, a_{6}{ }^{\prime} * b_{6}^{\prime}, a_{7}^{\prime} * b_{7}^{\prime}, a_{8}^{\prime} * b_{8}\right)
\end{gathered}
$$

\section{III.3. RANKING OF OCTAGONAL INTUITIONISTIC FUZZY NUMBERS}

The ranking function of Octagonal Intuitionistic Fuzzy Number (OIFN)

$\bar{A}_{o c}{ }^{I}=\left(a_{1}, a_{2}, a_{3}, a_{4}, a_{5}, a_{6}, a_{7}, a_{8}\right)\left(a_{1}{ }^{\prime}, a_{2}{ }^{\prime}, a_{3}{ }^{\prime}, a_{4}, a_{5}, a_{6}^{\prime}, a_{7}^{\prime}, a_{8}{ }^{\prime}\right)$ maps the set of all Fuzzy numbers to a set of real numbers defined as

$\mathrm{R}\left[\bar{A}_{o c}{ }^{I}\right]=\operatorname{Max}\left[\operatorname{Mag}_{\mu}\left(\bar{A}_{o c}{ }^{I}\right), \operatorname{Mag}_{\vartheta}\left(\bar{A}_{o c}{ }^{I}\right)\right]$ and similarly $R\left[\bar{B}_{o c}{ }^{I}\right]=\operatorname{Max}\left[\operatorname{Mag}_{\mu}\left(\bar{B}_{o c}{ }^{I}\right)\right.$, $\operatorname{Mag}_{\vartheta}\left(\bar{B}_{o c}{ }^{I}\right)$, Where

$$
\begin{array}{r}
\operatorname{Mag}_{\mu}\left(\bar{A}_{o c}{ }^{I}\right)=\frac{2 a_{1}+3 a_{2}+4 a_{3}+5 a_{4}+5 a_{5}+4 a_{6}+3 a_{7}+2 a_{8}}{28} \\
=\frac{2 a_{1}{ }^{\prime}+3 a_{2}{ }^{\prime}+4 a_{3}{ }^{\prime}+5 a_{4}+5 a_{5}+4 a_{6}{ }^{\prime}+3 a_{7}{ }^{\prime}+2 a_{8}{ }^{\prime}}{28}
\end{array} \quad \operatorname{Mag}_{\vartheta}\left(\bar{A}_{o c}{ }^{I}\right)
$$

\section{III.4. REMARK:}

If $\bar{A}_{o c}{ }^{I}$ and $\bar{B}_{o c}{ }^{I}$ are any two OIFNs. Then

1. $\bar{A}_{o c}{ }^{I}<\bar{B}_{o c}{ }^{I}$ if $\operatorname{Mag}_{\mu}\left(\bar{A}_{o c}{ }^{I}\right)<\operatorname{Mag}_{\mu}\left(\bar{B}_{o c}{ }^{I}\right)$ and $\operatorname{Mag}_{\vartheta}\left(\bar{A}_{o c}{ }^{I}\right)<\operatorname{Mag}_{\vartheta}\left(\bar{B}_{o c}{ }^{I}\right)$

2. $\quad \bar{A}_{o c}{ }^{I}>\bar{B}_{o c}{ }^{I}$ if $\operatorname{Mag}_{\mu}\left(\bar{A}_{o c}{ }^{I}\right)>\operatorname{Mag}_{\mu}\left(\bar{B}_{o c}{ }^{I}\right)$ and 
$\begin{array}{ll} & \operatorname{Mag}_{\vartheta}\left(\bar{A}_{o c}{ }^{I}\right)>\operatorname{Mag}_{\vartheta}\left(\bar{B}_{o c}{ }^{I}\right) \\ \text { 3. } & \bar{A}_{o c}{ }^{I}=\bar{B}_{o c}{ }^{I} \text { if } \operatorname{Mag}_{\mu}\left(\bar{A}_{o c}{ }^{I}\right)=\operatorname{Mag}_{\mu}\left(\bar{B}_{o c}{ }^{I}\right) \\ \text { 4. } & \operatorname{Mag}_{\vartheta}\left(\bar{A}_{o c}{ }^{I}\right)=\operatorname{Mag}_{\vartheta}\left(\bar{B}_{o c}{ }^{I}\right)\end{array}$

\section{III.5. MODI METHOD}

There are many methods to find the basic feasible solution, Modi method is heuristic method. The advantage of this method is that it gives an initial solution which is nearer to an optimal solution. Here in this paper Modi method is suitably modified and used to solving Intuitionistic Fuzzy transportation problem.

\section{Proposed Algorithm}

Step -1: In Octagonal Intuitionistic Fuzzy transportation problem (OIFN) the quantities are reduced into an integer using the ranking method called accuracy function.

Step - 2: For an initial basic feasible solution with $\mathrm{m}+\mathrm{n}-1$ occupied cell, calculate $u_{i}$ and $v_{j}$ for rows and columns. The initial solution can be obtained by any of the three methods discussed earlier.

To start with, any of $u_{i}$ 's or $v_{j}$ 's assigned the value zero. It is better to assign zero for a particular $u_{i}$ or $v_{j}$. Where there are maximum numbers of allocations in a row or column respectively, as it will reduce arithmetic work considerably. Then complete the calculation of $u_{i}$ 's and $v_{j}$ 's for other rows and columns by using the relation. $C_{i j}=u_{i}+v_{J}$ for all occupied cells (i,j).

Step - 3: For unoccupied cells, calculate opportunity cost by using the relationship $d_{i j}=C_{i j}-\left(u_{i}+v_{J}\right)$ for all iand $\mathrm{j}$.

Step - 4: Examine sign of each $d_{i j}$.

- If $d_{i j}>0$, then current basic feasible solution is optimal.

- If $d_{i j}=0$, then current basic feasible solution will remain unaffected but an alternative solutions exists.

- If one or more $d_{i j}<0$, then an improved solutions can be obtained by entering unoccupied cell $(\mathrm{i}, \mathrm{j})$ in the basis. An unoccupied cell having the largest negative value of $d_{i j}$ is chosen for entering into the solution mix (new transportation schedule).

Step - 5: Construct a closed path (or loop) for the unoccupied cell with largest negative opportunity cost. Start the closed path with the selected unoccupied cell and mark a plus sign $(+)$ in this cell, trace a path along the rows (or columns) to an occupied cell, mark the corner with minus sign (-) and continue down the column (or row) to an occupied cell and mark the corner with plus sign (+) and minus sign (-) alternatively, close the path back to the selected unoccupied cell.

Step - 6: Select the smallest quantity amongst the cells marked with minus sign on the corners of closed loop. Allocate this value to the selected unoccupied cell and add it to other occupied cells marked with plus signs and subtract it from the occupied cells marked with minus signs.

Step - 7: Obtain a new improved solution by allocating units to the unoccupied cell according to step -6 and calculate the new total transportation cost.

Step - 8: Test the revised solution further for optimality. The procedure terminates when all $d_{i j} \geq 0$, for unoccupied cells.

\section{III.6. NUMERICAL EXAMPLE:}

Consider Supplies and Demands are Octagonal Intuitionistic Fuzzy Number.

Table 1: To Find Octagonal Intuitionistic Fuzzy

\begin{tabular}{|c|c|c|c|c|}
\hline & $B_{1}$ & $B_{2}$ & $B_{3}$ & Supply \\
\hline$\overline{A_{1}}$ & 4.5 & 6.5 & 9.5 & $\begin{array}{l}(3,4,5,6,7,8,9,10) \\
(8,9,10,11,12,13,14,15)\end{array}$ \\
\hline$A_{2}$ & 7.5 & 11.5 & 8.5 & $\begin{array}{l}(3,4,5,6,7,8,9,10) \\
(6,7,8,9,10,11,12,13)\end{array}$ \\
\hline$A_{3}$ & 8.5 & 10.5 & 7.5 & $(1,2,3,5,6,7,8,13)$ \\
\hline
\end{tabular}




\begin{tabular}{|l|l|l|l|l|}
\hline & & & & $(0,1,2,3,4,7,9,10)$ \\
\hline Demand & $(7,8,9,10,11,12,13,14)$ & $(2,4,6,7,8,9,10,11)$ & $(1,2,3,5,6,7,8,10)$ & \\
& $(3,6,7,8,9,10,12,13)$ & $(1,2,3,4,5,6,7,10)$ & $(4,5,6,7,8,9,10,11)$ & \\
\hline
\end{tabular}

$\Sigma$ Demand $=\Sigma$ Supply

The problem is a balanced transportation problem. Using the proposed algorithm, the solution of the problem is as follows.Applying accuracy function on Octagonal Intuitionistic Fuzzy Number $[(1,2,3,4,5,6,7,8)(0,1,2,3,4,5,6,7)]$, we have

$\mathrm{R}\left(\bar{A}_{o c}{ }^{I}\right)=\operatorname{Max}\left[\operatorname{Mag}_{\mu}\left(\bar{A}_{o c}{ }^{I}\right), \operatorname{Mag}_{\vartheta}\left(\bar{A}_{o c}{ }^{I}\right)\right]$

$=\operatorname{Max}\left[\frac{2+6+12+20+25+24+21+16}{28}, \frac{0+3+8+15+20+20+18+14}{28}\right]$

$=\operatorname{Max}[4.5,3.5]$

$\mathrm{R}\left(\bar{A}_{o c}{ }^{l}\right)=4.5$

Similarly applying for all the values, we have the following table after ranking

Table 2: Reduced Table

\begin{tabular}{|l|l|l|l|l|}
\hline & \multicolumn{1}{|c|}{$B_{1}$} & \multicolumn{1}{c|}{$B_{2}$} & \multicolumn{1}{c|}{$B_{3}$} & Supply \\
\hline$A_{1}$ & 4.5 & 6.5 & 9.5 & 11.5 \\
\hline$A_{2}$ & 7.5 & 11.5 & 8.5 & 9.5 \\
\hline$A_{3}$ & 8.5 & 10.5 & 7.5 & 5.25 \\
\hline Demand & 10.5 & 7.25 & 8.5 & 26.25 \\
\hline
\end{tabular}

Applying VAM method, Table corresponding to initial basic feasible solution is

Table 3: Reduced Table of VAM Method

\begin{tabular}{|c|l|l|l|l|}
\hline & \multicolumn{1}{|c|}{$B_{1}$} & \multicolumn{1}{|c|}{$B_{2}$} & \multicolumn{1}{c|}{$B_{3}$} & Supply \\
\hline$A_{1}$ & {$[4.25]$} & {$[7.25]$} & 9.5 & \\
& 4.5 & 6.5 & 13.5 \\
\hline$A_{2}$ & {$[6.25]$} & 11.5 & 8.5 & \\
& 7.5 & 10.5 & $\begin{array}{l}5.25] \\
7.5\end{array}$ & 9.5 \\
\hline$A_{3}$ & 8.5 & 7.25 & & 5.25 \\
\hline \multirow{2}{*}{ Demand } & 10.5 & 8.5 & 26.25 \\
\hline
\end{tabular}

Since the number of occupied cell $m+n-1=5$ and are also independent. There exist non-negative basic feasible solutions.

The initial transportation cost is

$[(4.25 \times 4.5)+(7.25 \times 6.5)+(6.25 \times 7.5)+(3.25 \times 8.5)+(5.25 \times 7.5)]=180.125$

Applying MODI method, table corresponding to optimal solution is

Table 4: Reduced Table of MODI Method

\begin{tabular}{|c|c|c|c|c|c|}
\hline & $B_{1}$ & $B_{2}$ & 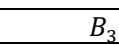 & Supply & $u_{i}$ \\
\hline$A_{1}$ & $\begin{array}{l}{[4.25]} \\
4.5\end{array}$ & $\begin{array}{l}77.25] \\
6.5\end{array}$ & $\begin{array}{l}\text { (4) } \\
9.5\end{array}$ & 11.5 & 0 \\
\hline$A_{2}$ & $\begin{array}{l}6.25] \\
7.5\end{array}$ & $\begin{array}{l}\text { (2) } \\
11.5\end{array}$ & $\begin{array}{l}{[3.25]} \\
8.5\end{array}$ & 9.5 & 3 \\
\hline$A_{3}$ & $\begin{array}{l}(2) \\
8.5\end{array}$ & $\begin{array}{l}(0.5) \\
10.5\end{array}$ & $\begin{array}{l}5.25] \\
7.5\end{array}$ & 5.25 & 2 \\
\hline Demand & 10.5 & 7.25 & 8.5 & 26.25 & \\
\hline$v_{j}$ & 4.5 & 6.5 & 5.5 & & \\
\hline
\end{tabular}

Since all $d_{i j} \geq 0$ the solution in optimum and unique.

The solution is given by $x_{11}=4.25$,

$x_{12}=7.25, x_{21}=6.25, x_{23}=3.25, x_{33}=5.25$

The optimal solution is

$=[(4.25 \times 4.5)+(7.25 \times 6.5)+(6.25 \times 7.5)+(3.25 \times 8.5)+(5.25 \times 7.5)]$

$=180.125$ 


\section{Conclusions}

In this paper, we discussed finding optimal solution for Octagonal Intuitionistic Fuzzy Transportation problem. Octagonal Intuitionistic Fuzzy numbers are apply in Supplies and Demands to easily coverted. We have used Accuracy function ranking method and Modi Method to easily understand and to arrive at nearer optimum solution.The transportation problems deal with assigning sources and jobs to destinations and machines. We will easily discuss the Transportation problem in Modi method. In future research we would propose generalized Octagonal Intuitionistic Fuzzy Numbers to deal problems and handling real life transportation problem having Intuitionistic Fuzzy Numbers.

\section{References}

[1] Fuzzy sets and K.Atanassov.1989. More on Intuitionistic Fuzzy sets, Fuzzy sets and systems, 33, pp.37-46.

[2] Atanassov .K.T. "Intuitionistic Fuzzy Sets", Fuzzy sets and systems, Vol.20 (1),pp: 87-96,(1986)

[3] A.Thamaraiselvi and R. Santhi, "On Intuitionistic Fuzzy Transportation Problem Using Hexagonal Intuitionistic Fuzzy Numbers", International Journal of Fuzzy Logic systems (IJFLS) Vol.5, No.1, January 2015.

[4] Thangaraj Beaula - M. Priyadharshini, “ A New Algorithm for Finding a Fuzzy Optimal Solution for Intuitionistic Fuzzy Transportation Problems, International Journalof Applications of Fuzzy Sets and Artificial Intelligence ( ISSN 2241-1240), Vol.5(2015),183- 192

[5] Dr.S.Ismail Mohideen, K.Prasanna Devi, M. Devi Durga, "Fuzzy Transportation Problem of Octagon Fuzzy Numbers with $\alpha$-Cut and Ranking Technique", Dr.Ismail Mohideen et al, Journal of Computer - JoC, Vol.1 Issue.2, July-2016, pg-60-67.

[6] Dr.Chandrasekaran,G.Gokila, Juno Saju, “ Ranking of Octagonal Fuzzy Numbers for Solving Multi Objective Fuzzy Linear Programming Problem with Simplex Method and Graphical Method, International Journal of Scientific Engineering and Applied Science (IJSEAS) - Volume -1, Issue-5, August-2015.

[7] Dr.M.S.Annie Christi Int. "Transportation Problem with Pentagonal Intuitionistic Fuzzy Numbers Solved Using Ranking Technique and Russell's Method, Journal of Engineering Research and Applications, ISSN: 2248 - 9622, Vol.6.Issue 2, (part-4), Feb 2016, pp.82-86.

[8] Nagoor Gani.A, Abbas. S,(2013) “A New method for solving in Fuzzy Transportation Problem”, Applied Mathematics Sciences, vol.7,No.28, pp.1357 - 1365

[9] O’heigeartaigh.H,(1982) “A Fuzzy Transportation Algorithm” Fuzzy Sets and Systems, pp.235-243. 